\title{
Coexistence of anti-MuSK antibody-positive myasthenia gravis and rheumatoid arthritis
}

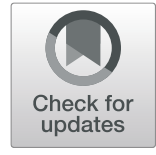

\author{
Amber Eker ${ }^{1^{*}}$ (D), Mehtap Tınazlı ${ }^{2}$ and Senem Ertugrul Mut $^{3}$
}

\begin{abstract}
Background: Rheumatoid arthritis (RA) is an autoimmune disease characterized with symmetric synovitis and occasional extra articular involvement; also, some neurologic disorders can be observed during the disease course. Myasthenia gravis (MG) is one of them and it is an autoimmune disease of neuromuscular junction. It is caused by autoantibodies against neuromuscular junction proteins: the nicotinic acetylcholine receptor (AChR) and the muscle specific tyrosine kinase (MuSK). Very few studies have reported the associated autoimmune disorders in MuSK-MG. Here, we present the first patient who has MuSK antibody-positive MG gravis and rheumatoid arthritis.

Case presentation: A 53-year-old woman with RA presented with fatigue and fluctuating proximal muscle weakness. Her electroneurophysiological investigation resembled MG. Her AchR antibody level was normal but MuSK antibodies were high. After the acute treatment with plasmapheresis which lead to complete recovery in myasthenic symptoms, she is following with mycophenolate mofetil.
\end{abstract}

Conclusions: Concomitant autoimmune disorders are common in the population. MG should be considered in patients with an autoimmune disorder and developing new neuromuscular weakness.

Keywords: Myasthenia gravis, Anti-MuSK antibody, Rheumatoid arthritis

\section{Background}

It is reported that at least one or more autoimmune disorders may affect $5 \%$ of the population [1]. Rheumatoid arthritis (RA) is one of the most common autoimmune rheumatic diseases characterized with synovial inflammation and joint destruction. An external trigger may induce an autoimmune reaction, leading to this chronic joint inflammation and destruction and some extraarticular organ involvement, including the skin, eye, heart, lung, renal, gastrointestinal, and nervous systems [2]. Both central and peripheral nervous system involvement may be observed during the disease course. Neurological associations in rheumatic diseases should be distinguished from them [3]. Myasthenia gravis (MG) is one of these associations and it is an autoimmune disease of neuromuscular junction. Clinically, MG is characterized

\footnotetext{
* Correspondence: amber.eker@emu.edu.tr; ambereker@emu.edu.tr

${ }^{1}$ Faculty of Medicine Department of Neurology, Eastern Mediterranean

University, via Mersin 10 Famagusta, Northern Cyprus, Turkey

Full list of author information is available at the end of the article
}

by muscle weakness and rapid fatigue aggravated by exercise and relieved by rest. Anti-AChR autoantibodies are detected in about the $85-90 \%$ of MG patients and lead to impaired neuromuscular junction transmission. In the remaining MG patients, the second common antibody against at the neuromuscular junction is antiMuSK. MuSK's role is mediating AChR clustering at the postsynaptic membrane. MG patients with anti-MuSK antibodies are a distinct MG subgroup (MuSK-MG) with different clinical characteristics from MG patients with anti-AChR antibodies (AChR-MG) and different pathogenetic mechanisms. Most of the anti-MuSK antibodies are the non-complement-binding IgG4 subclass in contrast to anti-AChR antibodies which are complementbinding IgG1 [1, 4-7].

Here, we present a patient with her written consent who developed anti-MuSK antibody-positive myasthenia gravis after 10 years of follow-up and treatment with rheumatoid arthritis. 


\section{Case presentation}

A 53-year-old woman presented to the university hospital neurology department with fatigue and fluctuating proximal muscle weakness. In her past medical history, the patient had also described recurrent joint pain and morning stiffness especially in the metacarpophalangeal and proximal interphalangeal joints which lasted for up to $1 \mathrm{~h}$, for about 10 years. C-reactive protein (CRP) levels were elevated to $4.05 \mathrm{mg} / \mathrm{dl}$ (normal $<0.05 \mathrm{mg} / \mathrm{dl}$ ), and erythrocyte sedimentation rate (ESR) was $23 \mathrm{~mm} / \mathrm{h}$ (normal $<20 \mathrm{~mm} / \mathrm{h}$ ). Anti-nuclear antibodies (ANA) and antibodies to extractable nuclear antigens (ENA) were not identified. Rheumatoid factor (RF) and anti-cyclic citrullined peptide were all negative. Rheumatoid arthritis was diagnosed according to 2010 American College of Rheumatology/European League Against Rheumatism Classification Criteria for RA by using a combination of her clinical, laboratory, and imaging features [8]. She said that she only used methotrexate for her arthritis treatment until her recent admittance; the vital signs were normal. Her initial neurologic examination revealed only $4 / 5$ strength in both upper and lower extremities proximals. All cranial nerves were intact. No sensory impairment was noticed. Deep tendon reflexes were bilaterally normoactive in all areas. Babinski was negative. Her electroneurophysiological investigation with the Natus Synergy, Ireland, 2010 system showed significant decremental response in low frequencies in repetitive stimulation of left trapezius muscle (Fig. 1a). Stimulating single-fibre electromyography in right extensor digitorum communis revealed significant jitter which represents neuromuscular junction disorder (Fig. 1b). Her anti-acetylcholine receptor (AChR) antibody level was normal but anti-muscle-specific tyrosine kinase (MuSK) antibodies were high $(1.82 \mathrm{nmol} / \mathrm{L}$; normal < $0.05 \mathrm{nmol} / \mathrm{L}$ ). Her thorax computed tomography did not show any thymic pathology. Because of rapidly progression of the weakness to the neck flexors and bulbar muscles, we performed 5 sessions of plasmapheresis. After the acute treatment of the symptoms, we observed complete recovery in myasthenic symptoms and we started oral steroid which was reduced and switched by mycophenolate mofetil.

\section{Discussion}

Patients affected by one autoimmune disorder have a higher risk of developing a second one, and the prevalence is higher in females than in males. MG patients have an increased risk of other autoimmune disorders compared to the rest of the population without MG. Autoantibodies that are characteristic for autoimmune disorders can be found in MG patients without any of the clinical symptom [9]. Furthermore the prevalence of manifest autoimmune disorders in patients with MG has

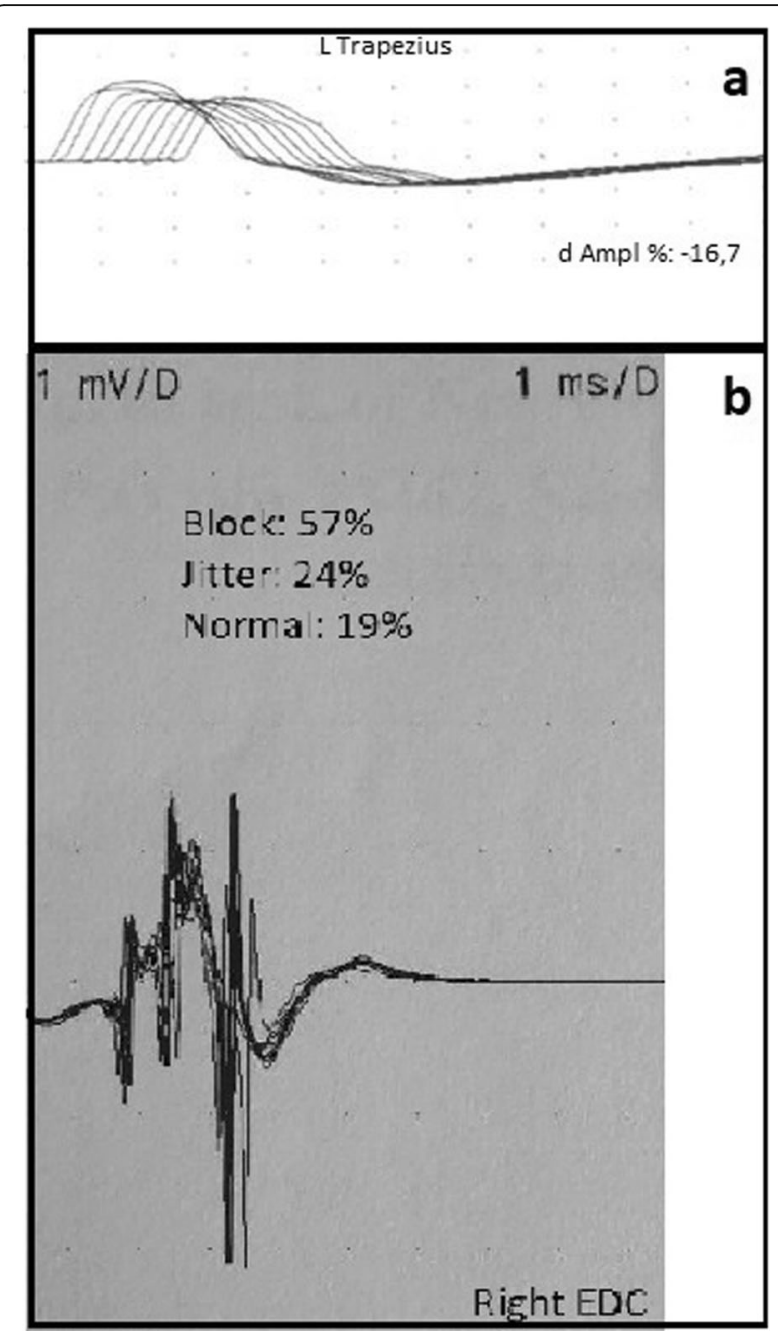

Fig. 1 Left trapezius muscle and right extensor digitorum communis

been reported in the ranges from 8.7 to $25 \%$ in the literature [4, 9-11]. In a systematic review, they stated that autoimmune thyroid disease was the most frequent autoimmune disorder, occurring in $10 \%$ of MG patients [9]. Other common autoimmune associates with MG are systemic lupus erythematosus (SLE), RA, dermatomyositis, polymyositis, and Addison's disease [1]. MuSK antibodies are present in 10-70\% of all MG patients without AChR antibodies. Only few studies have reported the associated autoimmune disorders in MuSK-MG. An association between MuSK-MG and SLE or relapsingremitting multiple sclerosis (MS) has been suggested in previous reports $[1,12,13]$.

Combined RA and MG occurrence has been calculated as $4 \%$ in a previous study with 75 MG patients [4]. From the literature review only one RA patient who is treated with penicillamine has been reported to have MuSK antibody positivity besides the AchR antibodies [7]. Our patient had not received any treatment with penicillamine which 
may become a triggering factor for her MG [14]. All the cases which have been reported in the literature have RA and AchR antibody-positive MG except the penicillaminerelated case $[4,7,15]$. To our knowledge, our case is the first reported case who has RA and anti-MuSK antibodypositive MG. If we concentrate also on the disease progression of co-occurred autoimmune disorder, it is reported that MG presentation was generalized in all the patients who have RA like our patient. Additionally, the manifestations of RA were also classically less severe [4].

The pathogenesis for the co-occurrence of different varieties of autoimmune disorders is unclear; however, genetic, infectious, and immunological factors have been implicated, and abnormalities in both humoral and cellmediated immunity have been described. Genetic studies on the susceptibility genes in autoimmune disorders reveal that is the most strong relationship at the human leukocyte antigen (HLA) locus [1, 4]. Particular role of the HLA-B8-DR3 and HLA DR14-DQ5 had been suggested in, respectively, MS and pemfigus association with MuSKMG [5, 16-18]. CTLA4 gene polymorphisms are also reported as associated with MG and other autoimmune diseases such as type 1 diabetes mellitus, autoimmune thyroid disease, SLE, RA, and celiac disease [1].

\section{Conclusion}

Similar environmental triggers in a genetically susceptible individual may lead to the co-occurrence of different autoimmune diseases in the same patient. Concomitant MG should be considered in patients with an autoimmune disorder and developing new neuromuscular weakness.

\section{Abbreviations \\ AChR: Acetylcholine receptor; ANA: Anti-nuclear antibodies; CRP: C-reactive protein; ENA: Extractable nuclear antigens; ESR: Erythrocyte sedimentation rate; HLA: Human leukocyte antigen; MG: Myasthenia gravis; MS: Multiple sclerosis; MuSK: Muscle specific tyrosine kinase; RA: Rheumatoid arthritis; RF: Rheumatoid factor; SLE: Systemic lupus erythematosus}

\section{Acknowledgements}

Not applicable

\section{Authors' contributions}

Conceptualization: AE and MT. Data curation: AE, MT, and SEM. Methodology: $A E, M T$, and SEM. Project administration: AE and MT. Resources: AE, MT, and SEM. Supervision: AE and MT. Writing-original draft: AE and MT. Writing - review and editing: AE, MT, and SEM. All authors have read and approved the manuscript

\section{Funding}

None

\section{Availability of data and materials}

Available from corresponding author on reasonable request

\section{Ethics approval and consent to participate}

Not applicable

\section{Consent for publication}

Written informed consent was obtained from the patient for publication of this case report.

\section{Competing interests}

The authors declare that they have no competing interests.

\section{Author details}

${ }^{1}$ Faculty of Medicine Department of Neurology, Eastern Mediterranean University, via Mersin 10 Famagusta, Northern Cyprus, Turkey. ${ }^{2}$ Faculty of Medicine Department of Internal Medicine, Near East University, Nicosia, Northern Cyprus, Turkey. ${ }^{3}$ Faculty of Medicine Department of Neurology, Kyrenia University, Karakum, Northern Cyprus, Turkey.

Received: 3 April 2020 Accepted: 14 August 2020

Published online: 28 August 2020

\section{References}

1. Nacu A, Andersen JB, Lisnic V, Owe JF, Gilhus NE. Complicating autoimmune diseases in myasthenia gravis: a review. Autoimmunity. 2015; 48(6):362-8.

2. Cojocaru M, Cojocaru IM, Silosi I, Vrabie CD, Tanasescu R. Extra-articular manifestations in rheumatoid arthritis. Maedica (Buchar). 2010;5(4):286-91.

3. Sofat N, Malik O, Higgens CS. Neurological involvement in patients with rheumatic disease. QJM. 2006;99(2):69-79.

4. Tamer S, Gokce Gunes HN, Gokcal E, Yoldas TK. Coexistence of autoimmune diseases and autoantibodies in patients with myasthenia gravis. Neurol India. 2016;64:45-9.

5. McConville J, Farrugia ME, Beeson D, Kishore U, Metcalfe R, Newsom-Davis J, et al. Detection and characterization of MuSK antibodies in seronegative myasthenia gravis. Ann Neurol. 2004;55(4):580-4.

6. Tsiamalos P, Kordas G, Kokla A, Poulas K, Tzartos SJ. Epidemiological and immunological profile of muscle-specific kinase myasthenia gravis in Greece. Eur J Neurol. 2009;16(8):925-30.

7. Poulas K, Koutsouraki E, Kordas G, Kokla A, Tzartos SJ. Anti-MuSK- and antiAChR positive myasthenia gravis induced by d-penicillamine. J Neuroimmunol. 2012;250:94-8.

8. Aletaha D, Neogi T, Silman AJ, Funovits J, Felson DT, Bingham CO 3rd, et al. Rheumatoid arthritis classification criteria: an American College of Rheumatology/European League Against Rheumatism collaborative initiative. Arthritis Rheum. 2010;62(9):2569-81.

9. Mao ZF, Yang LX, Mo XA, Qin C, Lai YR, He NY, et al. Frequency of autoimmune diseases in myasthenia gravis: a systematic review. Int J Neurosci. 2011;121:121-9.

10. Fang F, Sveinsson O, Thormar G, Granqvist M, Askling J, Lundberg IE, et al. The autoimmune spectrum of myasthenia gravis: a Swedish populationbased study. J. Intern. Med. 2014;277:594-604.

11. Christensen PB, Jensen TS, Tsiropoulos I, Sørensen T, Kjaer M, HøjerPedersen $\mathrm{E}$, et al. Associated autoimmune diseases in myasthenia gravis. A population-based study. Acta Neurol. Scand. 1995;91:192-5.

12. Nakamura H, Usa T, Motomura M, Ichikawa T, Nakao K, Kawasaki E, et al. Prevalence of interrelated autoantibodies in thyroid diseases and autoimmune disorders. J. Endocrinol. Invest. 2008;31:861-5.

13. Sylvester J, Purdie G, Slee M, Gray JX, Burnet S, Koblar S. Muscle-specific kinase antibody positive myaesthenia gravis and multiple sclerosis copresentation: a case report and literature review. J. Neuroimmunol. 2013; 264:130-3.

14. Schiavo AL, Guerrera V, Migliaresi S, Lombardi ML, Ruocco V. Coexistence of rheumatoid arthritis, myasthenia gravis, and pemphigus superficialis. JEADV. 1995;5(2):191-4.

15. Chai JY, Jeon CH, Cha HS, Kim BJ, Koh EM. 4 cases of myasthenia gravis in patients with rheumatoid arthritis. Korean J Intern Med. 2006;71(3):1147-52.

16. Fang F, Sveinsson $\mathrm{O}$, Thormar $\mathrm{G}$, Grangvist M, Askling J, Lundberg IE, et al. The autoimmune spectrum of myasthenia gravis: a Swedish populationbased study. J Intern Med. 2015:277(5):594-604.

17. Niks EH, Kuks JB, Roep BO, Haasnoot GW, Verduijn W, Ballieux BE, et al. Strong association of MuSK antibody-positive myasthenia gravis and HLADR14-DQ5. Neurology. 2006;66:1772-4.

18. Niks EH, Kuks JB, Verschuuren JJ. Epidemiology of myasthenia gravis with anti muscle specific kinase antibodies in The Netherlands. J. Neurol. Neurosurg. Psychiatry. 2007;78:417-8.

\section{Publisher's Note}

Springer Nature remains neutral with regard to jurisdictional claims in published maps and institutional affiliations. 